\title{
भारतीय अर्थव्यवस्थेची वाटचाल- एक अध्ययन (2014 ते 2020)
}

\author{
डॉ. रक्षित मदन बागडे,
}

(Dr. Rakshit Madan Bagde)

सहाय्यक प्राध्यापक, स्व. मन्सारामजी पडोके कला महाविद्यालय, गणेशपुर, भंडारा

ORCID iD - 0000-0002-7507-0244

Web of Science ResearcherID - AAF-2760-2020

SSRN - Author ID- 4770534

Authenticus ID - R-00J-YM2

rakshitbagde@gmail.com

सार

जीडीपीमधील उद्योगातील वाटा स्थिर असला तरी त्यात लक्षणीय मूलभूत परिवर्तन झाले. या कालावधीत उत्पादन पुनर्गठनाची प्रक्रिया म्हणून, सकल मूल्य संयोजित असताना उत्पादन सध्याच्या किंमतींवर वार्षिक 8 टक्क्यांनी वाढले होते. त्यानंतर 2004-09 मध्ये सकल मूल्यवर्धित वाढीचा वेग वाढून 20 टक्के झाला. याच किंमतींवर वार्षिक परंतु लक्षणीय म्हणजे रोजगारामध्येही वार्षिक 7.5 टक्क्यांनी वाढ झाली होती. 2009-10 मध्ये कामाच्या सहभागाचे प्रमाण 39.2 टक्के होते. त्यापैकी 53 टक्के शेतीमध्ये होते तर उर्वरित 47 टक्के बिगर कृषी क्षेत्रामध्ये होते. 2000 च्या उत्तरार्धात पहिल्यांदाच कृषी क्षेत्रातील परिपूर्ण कामगार संख्या घटली. एकूण अर्थव्यवस्थेत बेरोजगारीचे दरही 2004-05 मध्ये 8.3 टक्क्यांवरून घसरून 2009-10 मध्ये 6.6 टक्क्यांपर्यंत खाली आले होते. आपण असे म्हणू शकतो की 1991 नंतर भारतीय अर्थव्यवस्थेने चांगली कामगिरी केली पण सध्या भारतीय अर्थव्यवस्था दुसर्या अशांत अवस्थेतून जात आहे. सण 2014 पासून भारतीय अर्थव्यवस्थेचा वाढीचा दर कमी कमी होतो आहे. यात भर म्हणून कोविड 19 ने भारतात पाय पसरून विकास दराला खीळ लावली आहे. संबंधित शोध निबंधातून सण 2014 ते 2020 या कालखंडातील भारतीय अर्थव्यवस्थेची वाटचाल कशी आहे, तसेच तीन आर्थिक क्षेत्राचे अध्ययन करून निष्कर्ष काढण्यात येणार आहे.

कीवर्ड: अर्थव्यवस्था, शेती, उद्योग, सेवा.

\section{प्रस्तावना}

1991 पासून भारताने नियोजित अर्थव्यवस्था म्हणून सामान्यतः भरभराट केली आहे. पहिल्या काही पंचवार्षिक योजना ज्या विकासावर केंद्रित होत्या त्यात भारतीय अर्थव्यवस्थेचा कणा बनविण्यासाठी उत्पादन व औद्योगिक क्षेत्राला बळकटी आणणे तसेच शेती, दारिद्य निर्मूलन, रोजगार निर्मिती, सामाजिक विकास यावर भर देण्यात आला. इ.स. 1991 मध्ये, भारताने आतापर्यंतच्या सर्वात गंभीर चलन संकटाशी सामना केला होता, परंतु नंतर आर्थिक सुधारणा आणि एलपीजी (उदारीकरण, खाजगीकरण आणि जागतिकीकरण) चे धोरण अवलंबिले. 1990 च्या दशकात आर्थिक सुधारणांमुळे सुरुवातीला भारत हळूहळू विकास करताना दिसला. वास्तविक जीडीपी वाढ 1990 च्या दशकात सरासरी 7.7 टक्के वरून 2000 च्या दशकात वाढून 7.3 टक्के पर्यंत वाढलेली दिसून येते. या कालावधीत विकास वाढीच्या प्रवेगाचे वैशिष्ट्य म्हणजे उद्योग आणि सेवांच्या विकास दरात वाढ होऊन शेती विकास दरात घट झालेली होती. 1960 च्या दशकाच्या मध्यातील “हरितक्रांती" मुळे ज्यात 
विशेषत: तृणधान्यांच्या उत्पादनात मोठ्या प्रमाणात वाढ झाली होती. नंतरच्या काळात यात कोणतीही उल्लेखनीय अशी तांत्रिक प्रगती झाली नव्हती. त्यामुळे 1990 च्या दशकात, "हरित क्रांती" ची गती मंदावली होती. 2000 च्या दशकात वार्षिक सरासरी जीडीपी वाढीसह विकासाच्या प्रक्षेपणाचा मुख्य प्रवाह घसरला. 2004-08 या 5 वर्षांच्या कालावधीसाठी विकास दर 9 टक्के होता. या काळात शेतीसह अर्थव्यवस्थेच्या सर्व उप-क्षेत्रांमध्ये जागतिक आर्थिक संकटामुळे वाढ प्रक्रियेत व्यत्यय निर्माण झाला. त्यानंतर 2009-11 मध्ये सरासरी वाढ घसरली आणि अर्थव्यवस्थेत लक्षणीय मंदी आली. वित्तीय वर्ष 2001 मध्ये जीडीपी वाढीतील मंदी मुख्यत्वे उच्च व्याज दर, महागाई आणि औद्योगिक उत्पादनात महत्त्वपूर्ण आकुंचन यामुळे निर्माण झाली. 1990 च्या दशकात 24.4 टक्के असलेला कृषी विकास दर 2009-11 मध्ये सुमारे 15 टक्के वर आला. याच काळात बांधकामासह सेवां क्षेत्राचा वाटा 52 टक्क्यांवरून 65 टक्क्यां पर्यंत वाढला. तथापि, चिंतेची बाब म्हणजे जीडीपीच्या सुमारे 20 टक्क्यांवर उद्योगाचा वाटा अपरिवर्तित राहिला आहे. हे असे सूचित करते की गेल्या दोन दशकांतील भारताच्या विकासाच्या वाढीचा वेग सेवा क्षेत्रावर अवलंबून आहे.

जीडीपीमधील उद्योगातील वाटा स्थिर असला तरी त्यात लक्षणीय मूलभूत परिवर्तन झाले. या कालावधीत उत्पादन पुनर्गठनाची प्रक्रिया म्हणून, सकल मूल्य संयोजित असताना उत्पादन सध्याच्या किंमतींवर वार्षिक 8 टक्क्यांनी वाढले होते. त्यानंतर 2004-09 मध्ये सकल मूल्यवर्धित वाढीचा वेग वाढून 20 टक्के झाला. याच किंमतींवर वार्षिक परंतु लक्षणीय म्हणजे रोजगारामध्येही वार्षिक 7.5 टक्क्यांनी वाढ झाली होती. 2009-10 मध्ये कामाच्या सहभागाचे प्रमाण 39.2 टक्के होते. त्यापैकी 53 टक्के शेतीमध्ये होते तर उर्वरित 47 टक्के बिगर कृषी क्षेत्रामध्ये होते. 2000 च्या उत्तरार्धात पहिल्यांदाच कृषी क्षेत्रातील परिपूर्ण कामगार संख्या घटली. एकूण अर्थव्यवस्थेत बेरोजगारीचे दरही 2004-05 मध्ये 8.3 टक्क्यांवरून घसरून 2009-10 मध्ये 6.6 टक्क्यांपर्यंत खाली आले होते.

आपण असे म्हणू शकतो की 1991 नंतर भारतीय अर्थव्यवस्थेने चांगली कामगिरी केली पण सध्या भारतीय अर्थव्यवस्था दुसर्या अशांत अवस्थेतून जात आहे. सण 2014 पासून भारतीय अर्थव्यवस्थेचा वाढीचा दर कमी कमी होतो आहे. यात भर म्हणून कोविड 19 ने भारतात पाय पसरून विकास दराला खीळ लावली आहे. संबंधित शोध निबंधातून सण 2014 ते 2020 या कालखंडातील भारतीय अर्थव्यवस्थेची वाटचाल कशी आहे, तसेच तीन आर्थिक क्षेत्राचे अध्ययन करून निष्कर्ष काढण्यात येणार आहे.

की-शब्द - अर्थव्यवस्था, शेती, उद्योग, सेवा.

संशोधन पद्धती - संबंधित अध्ययनाकरिता दुय्यम साधनांचा वापर करण्यात येणार आहे. त्याकरिता विविध प्रकाशित लेख आणि अहवालांचा आधार घेण्यात येईल.

\section{संशोधन साहित्याचा आढावा -}

1) Namrata Anand-An Overview of Indian Economy (1991-2013) प्रस्तुत शोध लेखात नवीन आर्थिक सुधारणेपासूनच्या आर्थिक परिस्थितीचा आढावा घेतलेला आहे. त्यात आर्थिक धोरणांचा चांगला प्रभाव अर्थव्यवस्थेवर पडला असल्याचे निष्कर्षातून दिसून येते. या सुधारणेमुळे वित्तीय तूट कमी होत असल्याचे दिसून येते.

2) विक्रान्त सिंह- क्या बेहाल अर्थव्यवस्था की 2020 में बदलेगी सूरत? सन 2019 च्या शेवटच्या टप्यावरून आपण असा निष्कर्ष काढू शकतो की येणारी वेळ फक्त भारताची आहे. परंतु यासाठी सुस्त भारतीय अर्थव्यवस्था वाचविणे आवश्यक आहे. जर सध्या चालू असलेली आर्थिक सुस्ती आर्थिक मंदीमध्ये बदलली तर लक्षात ठेवा की कोणत्याही अर्थव्यवस्थेतील मंदीचा परिणाम नेहमीच हानिकारक ठरला आहे. 
3) ज़ुबैर अहमद- evఆp ग्रोथ रेट के हिसाब से भारतीय अर्थव्यवस्था बीते छह साल के सबसे निचले स्तर पर, या लेखातून 201920 च्या पहिल्या तिमाहीचा डेटा जारी केला आहे. त्यानुसार आर्थिक विकास दर 5 टक्के झाला आहे. मागील आर्थिक वर्षाच्या याच तिमाहीत विकास दर 8 टक्के होता. गेल्या तिमाहीत ही सर्वात कमी आणि मोदी सरकारच्या काळात सर्वात कमी वाढ होती. देशाच्या अर्थव्यवस्थेच्या विकासाची गती कमी होत असल्याचे तज्जांचे म्हणणे आहे. गेल्या तीन वर्षांपासून हे घडत आहे.

कृषी

भारतीय अर्थव्यवस्था 21 व्या शतकात सुद्धा आर्थिक विकासाच्या बाबतीत शेती क्षेत्रावर अवलंबून आहे. 31 जानेवारी, 2020 रोजी जाहीर झालेल्या आर्थिक सर्वेक्षण अहवाला नुसार शेतकर्यांच्या उत्पन्नावर होणान्या परिणामाच्या बदल्यात कृषी व त्याशी संबंधित क्षेत्रातील वास्तविक सरासरी वार्षिक विकास दर गेल्या सहा वर्षांत स्थिर राहिले आहे. सर्वेक्षणानुसार 2014-15 पासून ते 2018-19 या कालावधीत कृषी व त्याशी संबंधित क्षेत्रातील वार्षिक वाढीचा दर 2.8 टक्के होता. 2019-20 मध्ये अपेक्षित विकास दर 2.9 टक्के आहे. आर्थिक सर्वेक्षण अहवाल 2020 मध्ये, अल्पसंख्याक आणि अल्पभूधारक शेतक-यांना आधार देण्यासाठी टिकाऊ शेती पद्धतींच्या महत्वावर जोर देण्यात आला आहे, जे भारतातील 87 टक्के शेतकरी आहेत. त्यात डायनॅमिक पीक पद्धती, रासायनिक खतांचा न्याय्य वापर आणि कार्यक्षम सिंचन प्रणालींसह संसाधन कार्यक्षम पध्दतींचे संयोजन करण्याची आवश्यकता आहे. असे म्हटले आहे की मातीची विटंबना झाल्यामुळे अपुरी सिंचन प्रणाली आणि रासायनिक खतांचा अयोग्य वापर यामुळे कृषी उत्पादकतेवर परिणाम झाला.

कृषी विकास साध्य करण्या करिता पंतप्रधान किसान निधी (पीएम-किसान) योजना तत्कालीन केंद्रीय अर्थमंत्री पीयूष गोयल यांनी अंतरिम अर्थसंकल्प 2019-20 मध्ये जाहीर केली होती. 2019 च्या लोकसभा निवडणुकांच्या पूर्ववर्ती भारतीय जनता पक्षाच्या सरकारचा हा मास्टर स्ट्रोक सजला जात होता. 2011 ते 2015 या कालावधीत भारतीय शेतकर्यांच्या वास्तविक उत्पन्नात केवळ 0.44 टक्क्यांनी वाढ झाली असल्याचे शासकीय थिंक-टँक नीति आयोगाचे म्हणणे आहे. 2017 आणि 2018 मध्ये ही किमान वास्तविक वाढ 'शून्य' होती. याचा परिणाम म्हणून, सरकारने थेट उत्पत्र आधार योजना जाहीर केली, ज्यात एकूण 75,000 कोटींचे वाटप झाले. त्याअंतर्गत प्रत्येक शेतक्याला तीन हप्त्यांमध्ये वर्षाकाठी सहा हजार रुपये मिळतील. कृषी व व्यापार धोरण विश्लेषक दविंदर शर्मा यांच्यानुसार "2000 पासून भारतातील शेतक-यांचे उत्पन्न स्थिर आहे." सरकारने शेतकर्यांना थेट उत्पन्न आधार देऊन कौतुकास्पद पाऊल उचलले असले तरी, परिस्थितीत काही बदल घडवून आणला जाऊ शकला नाही. सरकारने प्रत्येक शेतक्याला वर्षाकाठी 18,000 रुपये देण्याचा विचार केला पाहिजे. आर्थिक सहकार आणि विकास संघटना आणि भारतीय संशोधन परिषदेच्या संयुक्त अभ्यासानुसार 2000-01 ते 2016-17 या कालावधीत शेतक-यांना 45 लाख कोटी रुपयांचे नुकसान झाले आहे, कारण भारतीय शेतक-्यांना त्यांच्या उत्पादनाच्या योग्य मोबदल्यापासून वंचित ठेवण्यात आले आहे. दुसर्या शब्दांत सांगायचे तर, शेतक-्यांना एका वर्षात 2.65 लाख कोटी रुपयांचे नुकसान झाले, मुख्यत: कमी किंमतीमुळे, आश्वासित खरेदीतील कपात, व्यापार धोरणांमध्ये कपात आणि विपणन धोरणांमध्ये मोठी कपात करण्यात आलेली आहे.

सरकारांच्या अर्धवट दृष्टीकोनामुळे मुख्यतः फारसा बदल झाला नाही. पंतप्रधान-किसान योजने अंतर्गत देखील 50 टक्के पेक्षा जास्त शेतकच्यांना योजनेचे तीन हप्ते मिळाले नाहीत. यात काहीच आश्चर्य नाही की शेतक्यांच्या आत्महत्या सुरूच आहेत. 2018 मध्ये नॅशनल क्राइम रेकॉर्ड ब्युरोने (एनसीआरबी) संपूर्ण भारत भरात अशा प्रकारच्या 1,03,349 शेतकच्यांच्या आत्महत्या नोंदवल्या आहेत. एनसीआरबीच्या म्मणण्यानुसार, 1995 ते 2018 या काळात भारतात झालेल्या आत्महत्यांची एकूण संख्या $3,53,802$ होती. यापैकी सुमारे 86 टक्के $(3,03,597)$ पुरुष आणि उर्वरित $(50,188)$ महिला होत्या. यातच भर म्हणून 2020 मध्ये भारतीय संसदेने प्रामुख्याने कृषी कायदा-2020 संसदेद्वारे पारित केल्याने तीन कृषी कायद्यांविरूद्ध पंजाब, 
हरियाणा, राजस्थान, छत्तीसगड आणि जवळपास संपूर्ण देशातील शेतकच्यांचे आंदोलन सुरु आहे. शेतकरी संघटणांनी नवीन धोरण ' हे 'शेतकरीविरोधी' आणि 'शेतीविरोधी' आहे असे वर्णन केले गेले आहे.

उद्योग -

भारताच्या औद्योगिक विकासाला चालना व गती पंचवार्षिक योजनांच्या काळात मिळाली आणि स्वयंचलित व स्वावलंबी औद्योगिक विकासाचा पाया घातला गेला. नियोजनात निश्चित केलेल्या दिशेने औद्योगिक विकास करण्याकरिता औद्योगिक धोरणास अनुसरून उद्योग धंद्यांच्या वाढीचे अग्रक्रम ठरविण्यात आले.

8 नोक्ेंबर 2016 रोजी मोदी सरकारने राबविलेल्या नोटाबंदीचा मोठ्या प्रमाणात व्यवसायांवर परिणाम झाला. विशेषत: छोट्या छोट्या व्यवसायांना त्रास सहन करावा लागला. 2016 नंतरच्या परिस्थितीत अनेक उद्योग क्षेत्रामध्ये मोठ्या प्रमाणात नोकरी गमावली आहे. जुलै 2017 मध्ये सरकारने जीएसटी (वस्तू व सेवा कर) देखील आणला ज्याला भाजपा सरकारने मोठ्या प्रमाणात सुधारणांचा विचार केला. कर लागू करण्याचे नियमन व पद्धतशीर करण्यासाठी हे पाऊल उचलून धरले असले तरी अंमलबजावणी तितकी सोपी नक्हती. निराकरण करण्यासाठी अनेक अडचणी आल्या आणि ही अंमलबजावणी हडबडी पद्धतीने झाली. ज्यामुळे जीडीपीची वाढ मंदावली. 2014 नंतर जीडीपी वाढ कमी होण्यास मदत करणारे आणखी एक क्षेत्र म्हणजे खाजगी गुंतवणूक विभाग.

25 सप्टेंबर, 2014 रोजी, भारत सरकारने मॅन्युफॅक्चरिंगला प्रोत्साहित करण्यासाठी 'मेक इन इंडिया' उपक्रम जाहीर केला आणि मॅन्युॅॅक्चरिंग आणि सर्क्तिसेसमध्ये समर्पित गुंतवणूकीने अर्थव्यवस्थेला गॅल्वनाइज केले. योजनेच्या घोषणे नंतर लगेचच कोटींच्या गुंतवणूकीची घोषणा केली गेली. 'मेक इन इंडिया' ही कल्पना नवीन नाही. कारखान्याच्या उत्पादनाचा देशामध्ये दीर्घ इतिहास आहे. या उपक्रमाने मात्र भारताला जागतिक उत्पादन केंद्र बनविण्याचे महत्त्वाकांक्षी लक्ष्य ठेवले आहे. हे लक्ष्य साध्य करण्यासाठी, लक्ष्य ओळखले गेले आणि धोरणे अधोरेखित केली. तीन प्रमुख उद्विष्ट्ये अशी (अ) अर्थव्यवस्थेतील क्षेत्राचा वाटा वाढविण्यासाठी उत्पादन क्षेत्राचा विकास दर वर्षाकाठी 12-14\% पर्यंत वाढवणे. (ब) 2022 पर्यंत अर्थव्यवस्थेत 100 दशलक्ष अतिरिक्त उत्पादन रोजगार निर्मिती; आणि (क) जीडीपीमध्ये उत्पादन क्षेत्राचे योगदान सध्याच्या 25 टक्क्यांवरून 2022 पर्यंत (सुधारित 2025 पर्यंत) 16\% केले जाईल याची खात्री करण्यासाठी. गुंतवणूकीसाठी अनुकूल वातावरण निर्माण करणे, आधुनिक व कार्यक्षम पायाभूत सुविधांचा विकास करणे आणि परकीय भांडवलासाठी नवीन क्षेत्रे उघडणे हा धोरणात्मक हष्टीकोन होता.

'मेक इन इंडिया' का अयशस्वी झाली? याची तीन कारणे आहेत. प्रथम, उत्पादन क्षेत्रातील उत्पादन साध्य करण्यासाठी महत्वाकांक्षी विकास दर ठरविन्यात आला. यात वार्षिक विकासाचा दर औद्योगिक क्षेत्राच्या क्षमतेपेक्षा चांगला असला तरी तो ऐतिहासिकहष्ट्या भारताने साध्य केला नाही. दुसरे म्हणजे, योजनेच्या पुढाकाराने बर्याच क्षेत्रांना आपल्या क्षेत्रात आणले. यामुळे मुख्य धोरणाकडे लक्ष कमी झाले. याउप्पर, हे देशांतर्गत अर्थव्यवस्थेच्या तुलनात्मक फायद्यांबद्दल कोणत्याही प्रकारचे धोरण म्हणून समजून न घेता फक्त धोरण म्हणून पाहिले गेले. तिसरे, जागतिक अर्थव्यवस्थेची अनिश्चितता आणि वाढता व्यापार संरक्षणवाद यांना पाहता हा उपक्रम नेत्रदीपक दुर्देवी ठरला.

सरकारने अनेक सार्वजनिक कंपन्यांच्या खासगीकरणाची प्रक्रिया सुरू केली आहे. एअर इंडिया - सरकारी मालकीची एअरलाइन्स, त्याशिवाय बीपीसीएल, इंडस्ट्रीयल डेव्हलपमेंट बँक ऑफ इंडिया (आयडीबीआय), आणि कंटेनर कॉप्पेरिशन ऑफ इंडिया (कॉन्कर) या कंपन्यांमध्ये केंद्र सरकारची हिस्सेदारी विक्री करण्याचे प्रस्ताव प्रलंबित आहेत. नव्या सुधारणांचा या निर्णयांवर परिणाम होईल की नाही हे अद्याप स्पष्ट झाले नाही. 
फेब्रुवारी 2020 मध्ये झालेल्या अर्थसंकल्पांच्या घोषणेदरम्यान, भारत सरकारने वित्तीय वर्ष 2021 पर्यंत निर्गुंतवणुकीतून 2.1 लाख कोटी मिळविण्याचे लक्ष्य ठेवले होते. मागील आर्थिक वर्षानंतरही सरकारने आपले लक्ष्य 1.05 लाख कोटी रुपयांवरून 65,000 कोटींवर नेले होते.

2017-18 दरम्यान भारतातील बेरोजगारीचा दर वाढून 45 वर्षांच्या उच्चांकावर पोचला असल्याचे सरकारी सर्वेक्षणात नमूद करण्यात आले आहे, असे बिझिनेस स्टँडर्ड वृत्तपत्राने नमूद के ले आहे. जुलै 2017 ते जून 2018 या कालावधीत झालेल्या राष्ट्रीय नमुना सर्वेक्षण कार्यालयाच्या अहवालात बेरोजगारीचा दर 6.1 टक्के असल्याचे दिसून आले आहे, जे 1972-73 नंतरचे सर्वात जास्त आहे. ग्रामीण भागातील 5.3 च्या तुलनेत शहरी भागात बेरोजगारी 7.8 टक्के आहे. आकडेवारी लक्षणीय आहे कारण नोक्हेंबर 2016 मध्ये सरकारने देशातील बहुतेक नोटा रात्रीतूनच मागे घेण्याच्या निर्णयानंतर घेतल्या गेलेल्या भारताच्या रोजगाराच्या परिस्थितीचे हे पहिले व्यापक मूल्यांकन होते.

2014 मध्ये अर्थव्यवस्थेचे औचित्य साधून आणि लाखो तरुणांना रोजगाराच्या- नोकरीच्या बाजारात प्रवेश करण्याच्या रोजगाराच्या संधींना वाव देण्याचे वचन देऊन मोदी सरकार सत्तेत आले. परंतु भारताची अर्थव्यवस्था दरवर्षी 7 टक्क्यांनी वाढत असताना - प्रमुख अर्थव्यवस्थां मध्ये वेगवान असमान वाढीचा अर्थ असा आहे की, वेगवान राहण्यासाठी पुरेशी नवीन रोजगार शिल्लक नाहीत. सरकारचे आर्थिक यशाचे दावे पोकळ वाटू लागले आहेत. सेंटर फॉर मॉनिटरिंग इंडियन इकॉनॉमी, एक अग्रगण्य स्वतंत्र थिंक-टँक म्हणाले की, गेल्या वर्षी देशात तब्बल 11 दशलक्ष रोजगार गमावले आहेत.

\section{सेवा क्षेत्र}

सेवा क्षेत्रामध्ये विमा, सरकार, पर्यटन, बँकिंग, किरकोळ, शिक्षण आणि सामाजिक सेवा या अर्थव्यवस्थेचे मऊ भाग असतात. सध्या सेवा क्षेत्र भारतीय जीडीपीच्या जवळपास $60 \%$ योगदान देत आहे. सेवा क्षेत्र तृतीयक क्षेत्र म्हणून देखील ओळखले जाते. भारतीय उद्योग परिसंघ संस्था (सीआयआय) आणि केपीएमजी यांनी प्रकाशित के लेल्या अहवालात म्हटले आहे की भारत सध्या जगातील सर्वात वेगवान सेवा अर्थव्यवस्था आहे. 2015-16 या आर्थिक वर्षात विकृतीच्या व्यापारामुळे सरकार सेवांच्या व्यापारावर मोठी बाजी मारत आहे. योगायोगाने, 2014-15 मध्ये जागतिक सेवा निर्यातीत भारताचा वाटा 3.2 टक्के होता, तो जागतिक व्यापार निर्यातीत व्यापार निर्यातीत दुपटीने 1.7 टक्के होता. जग सध्याच्या सेवांच्या दहा निर्यातदारांमध्ये भारत आठव्या स्थानावर आहे. माहिती तंत्रज्ञान ज्यामध्ये देश जागतिक क्रमांकाचा नेता आहे, गेल्या आर्थिक वर्षात 108 अब्ज डॉलर्सच्या सेवांच्या निर्यातीची नोंद झाली असून ती मुख्यत्वे युनायटेड स्टेट्स, युनायटेड किंगडम आणि युरोपमध्ये निर्यात केली गेली.

हे क्षेत्र देखील भारतातील सर्वात मोठे खाजगी क्षेत्रातील नियोक्ता आहे आणि त्यामध्ये 3.7 दशलक्षाहूनही अधिक लोक कार्यरत आहेत. 2016 मध्ये हा उद्योग 8.5 टक्क्यांपर्यंत वाढण्याचा अंदाज आहे.

मेक इन इंडिया' मोहिमेद्वारे उत्पादनाला जोरदार धक्का दिल्यानंतर पंतप्रधान नरेंद्र मोदी यांनी लवकरच देशाच्या जीडीपीच्या $60 \%$ क्षेत्राच्या सेवा क्षेत्रासारखेच काहीतरी करतील अशी अपेक्षा होती. राष्ट्रीय सांख्यिकी कार्यालयाने (एनएसओ) अंदाज व्यक्त केला आहे की एकूण जीव्हीए आर्थिक वर्ष 2021 मधील उणे 7.2 टक्क्यांपर्यंत घटेल (जीडीपी घटून वजा 7.7\% होईल). कृषी-जीव्हीए आणि युटिलिटीज-जीव्हीए (उद्योगाचा घटक) सकारात्मक वाढ नोंदवेल (अनुक्रमे $3 \%$ आणि $2.7 \%$ ) उत्पादन-जीव्हीए (उद्योगातील मुख्य घटक) उणे $12.4 \%$ ने कमी होईल. 'फायनान्शियल, रियल इस्टेट आणि प्रोफेशनल सर्क्तिसेस' चे जीव्हीए उणे $0.8 \%$, 'लोक प्रशासन, संरक्षण आणि इतर सेवा' उणे $3.7 \%$, 'बांधकाम' उणे $12.6 \%$ आणि 'व्यापार, हॉटेल, वाहतूक, संप्रेषण' व प्रसारण उणे $21 \%$ पर्यंत (सर्व स्थिर किंमतीवर). 


\begin{tabular}{|c|c|}
\hline वर्ष & GDP \% \\
\hline 2014 & 6.18 \\
\hline 2015 & 6.79 \\
\hline 2016 & 7.08 \\
\hline 2017 & 5.66 \\
\hline 2018 & 5.43 \\
\hline 2019 & 2.99 \\
\hline 2020 & -8.87 \\
\hline
\end{tabular}

\section{समारोप}

एकूणच सण 2014 पासून भारतीय अर्थव्यवस्था ही एक चिंतेची बाब बनली आहे. यात दिवसेंदिवस होत असलेले खाजगीकरण आणि खाजगी क्षेत्रावरचे अवलंबित्व याचा भविष्यात काय परिणाम होणार याची चाहूल यायला लागली आहे. कमी होत असलेली रोजगाराची पातळी, उत्पत्राची असमानता, दारिद्रय, उपासमार, मोठया प्रमाणात होत असलेले स्थलांतरण, राहणीमाच्या दर्जात होत असलेली घट, महागाई ची वाढ, शेतीची अनास्था या आणि अन्य अनेक आघाडीवर होत असलेली अर्थव्यवस्थेची पीछेहाट आपल्याला भविष्यात चिंतेची बाब नक्कीच ठरणार आहे.

\section{संदर्भ}

[1] Namrata Anand, An Overview of Indian Economy (1991-2013) IOSR Journal of Economics and Finance (IOSR-JEF) e-ISSN: 2321-5933, p-ISSN: 2321-5925.Volume 3, Issue 3. (Mar-Apr. 2014), PP 19-24 www.iosrjournals.org

[2] विक्रान्त सिंह, क्या बेहाल अर्थव्यवस्था की 2020 में बदलेगी सूरत?

[3] ज़ुबैर अहमद- GDP ग्रोथ रेट के हिसाब से भारतीय अर्थव्यवस्था बीते छह साल के सबसे निचले स्तर पर

[4] https://en.wikipedia.org/wiki/Economic_development_in_India

[5] https://www.firstpost.com/business/25-years-of-liberalisation-a-glimpse-of-indias-growth-in-14charts-2877654.html

[6] https://www.macrotrends.net/countries/IND/india/gdp-growth-rate

[7] https://www.business-standard.com/article/economy-policy/world-bank-sees-india-s-gdp-growth-at1-5-2-8-lowest-since-1991-crisis-120041200996_1.html

[8] https://economictimes.indiatimes.com/hindi/wealth/personal-finance/will-face-of-the-troubledeconomy-change-in-2020/articleshow/73047280.cms .

[9] https://www.downtoearth.org.in/news/agriculture/economic-survey-2019-20-agriculture-growthstagnant-in-last-6-years-69076

[10] https://www.businessinsider.in/politics/policy/news/india-will-merge-some-government-companiesand-privatise-some-cpse/articleshow/75785709.cms

[11] https://auto.economictimes.indiatimes.com/news/industry/indias-unemployment-rate-hit-45-yearhigh-in-2017-18-report/67772184

[12] https://www.businesstoday.in/opinion/columns/story/indian-economy-the-untold-story-of-indiasservices-sector-despite-being-main-driver-of-countrys-growth-story-284311-2021-01-13 